\title{
Wake up, South Africa! The antibiotic 'horse' has bolted
}

Decades of poor medical and veterinary antibiotic prescribing and a lack of regard for the practice of infection prevention and control (IPC) in our hospitals have left South Africa, like the rest of the international community, on the brink of a return to an era of untreatable bacterial infection. The recent emergence in South Africa of bacteria carrying the highly mobile New Delhi metallo- $\beta$ lactamase-1 (NDM-1) gene, ${ }^{1}$ which has been associated with rapid spread of carbapenem-resistant Enterobacteriaceae (CRE), and, for the first time in Africa, Klebsiella pneumoniae carbapenemases (KPCs), ${ }^{2}$ will have a profound effect on the lives of our patients and on the health service. The acquisition of drug-resistant hospitalacquired infections (HAIs) increases morbidity, mortality and the cost of patient management to an already beleaguered health system by increasing the duration of hospital stay, often in expensive intensive care units (ICUs), and antibiotic prescribing costs. ${ }^{3,4}$

Unlike the case of multidrug-resistant (MDR) and extensively drug-resistant tuberculosis, the situation we find ourselves in with MDR Gram-negative bacteria such as CRE cannot be blamed on poor patient compliance, or merely on the introduction of resistant strains from foreign climes. Rather, this is a home-grown problem, generated and perpetuated by doctors, nurses and allied healthcare workers in South Africa.

For resistant Gram-positive bacteria, there often remain more expensive antibiotic options. Vancomycin or teicoplanin are available to treat methicillin-resistant Staphylococcus aureus (MRSA), while linezolid or daptomycin may be used for MRSA as well as vancomycinintermediate $S$. aureus and vancomycin-resistant $S$. aureus. However, the situation for managing CRE is far more serious. We have become reliant on colistin, a nephrotoxic polymixin antibiotic developed in the 1960s. Reports of colistin-resistant Pseudomonas aeruginosa, K. pneumoniae and Acinetobacter baumannii rendering the bacteria pan-drug resistant are already documented. ${ }^{5-7}$ Most worrying of all is the total lack of new antibiotics against Gram-negative infections in the antibiotic pipeline for the next 10 - 20 years.

In this issue of the journal, Brink et al. ${ }^{8}$ set out the risk factors for acquisition of CRE and some of the issues around prevention and management. But how are we to respond to the wider issues of antibiotic stewardship in South Africa at this late stage, even though the 'horse has bolted'? The approach must be multifaceted. Simply put, we must force a return to rational antibiotic prescribing through strong antibiotic stewardship, with equal emphasis on addressing IPC to prevent spread of already resistant bacteria between patients.

IPC in South Africa lurches from crisis to crisis without getting to the core of the problem, that of collective responsibility. IPC is often viewed as the domain of a select few practitioners, mostly nurses, supported by microbiologists or infectious disease specialists, who are meant to police our hospitals and ensure that our patients and staff protect themselves and others from spreading and contracting communicable diseases. However, if we are to confront the crisis we find ourselves in and prevail, the first change required is in our mindset. IPC is not the domain of the few, but the responsibility of all. We must move towards a situation where all healthcare providers in this country become advocates for infection prevention and control, and practise what they preach. Trained IPC officers certainly have a role to play in governance of IPC, and government must meet its obligations to fund posts for IPC officers to meet standard norms. However, no number of IPC officers in a hospital can prevent the spread of MDR bacteria, if healthcare workers fail to disinfect their hands between patients. And here lies the one real hope for the future in terms of IPC. The changes that need to be put in place are simple. They do not require complex systems or costly financial interventions. There are few unknowns in the field, and research and clinical findings from other countries are equally relevant in our setting. Each healthcare institution in this country must have administrative and environmental policies in place to enable them to correctly identify and isolate patients with MDR bacterial infections and must provide running water, soap and hand disinfectants as well as the personal protective equipment required to ensure standard precautions against spread. The adoption of evidence-based, simple bundles of care designed by the Best Care ... Always! campaign ${ }^{9,10}$ simplifies the practice of IPC in the ICU and ward setting, and their adoption nationally would be another milestone for change.

Unlike IPC, the drivers for change in antibiotic prescribing are less simple. Rational antibiotic prescribing requires a basic knowledge of the mechanism of action of antibiotics, the leading causes of a particular infection, and the resistance patterns of bacteria in the local healthcare setting. Outside of central and private hospitals, surveillance of antibiotic resistance is rarely practised, hampering rational prescribing. Antibiotic stewardship refers to a multifaceted approach that healthcare organisations undertake to optimise prescribing, including policies, guidelines, surveillance, education and audit. ${ }^{11}$ In some private and public hospitals, antibiotic stewardship programmes (ASPs) are under way or being developed. To help co-ordinate these efforts across the healthcare sector, the Federation of Infectious Diseases Societies of Southern Africa (FIDSSA), which represents adult and paediatric infectious disease physicians, clinical microbiologists, and infection control, travel medicine and sexually transmitted diseases practitioners in southern Africa, arranged the first South African Antibiotic Stewardship Programme (SAASP) conference in February 2012. A working group developed an ASP for South Africa, the goals of which are to:

- Provide leadership, advocacy for and strengthening of antibiotic stewardship in the public and private sectors in South Africa

- Direct appropriate training in antibiotic stewardship and co-ordinate dissemination of antibiotic stewardship information to all sectors of South Africa's healthcare system and civil society

- Harmonise and unify existing national antibiotic prescribing guidelines and develop guidelines for those infections not already covered, for adult and paediatric practice, incorporating principles of antibiotic stewardship and optimal diagnostic testing

- Identify gaps in current knowledge and the necessary operational research/audit that will inform practice, and feed back the results of these studies to stakeholders, so as to implement change

- Engage with the National Department of Health and industry to address the economic issues and systemic obstacles surrounding antibiotic costs and stewardship.

As is the case for promotion of IPC, a move towards rational antibiotic prescribing must be embraced by all prescribers in South Africa and requires a commitment to education. The rise of CRE did not occur as a result of the injudicious use of carbapenems alone, but due to misuse and overuse of all antibiotics. We need to reduce the overall consumption of antibiotics, and to limit the duration of therapy in accordance with evidence-based guidelines when available, or expert opinion when not. We need to know what we are treating and to be able to de-escalate from broad-spectrum empiric therapy to targeted narrow-spectrum antibiotics that are less likely to lead to resistance. Taking appropriate cultures from sterile sites is therefore a key component of rational prescribing and a practice 
that is sorely lacking at all levels of healthcare in South Africa. Daily review of the route of administration of antibiotics is another key component, allowing a reduction in intravenous therapy and removal of indwelling intravascular catheters, thereby reducing the risk of catheter-associated bloodstream infections.

The horse has indeed bolted, but it is not too late to limit the emergence and spread of MDR bacterial infection in South Africa. To achieve this goal, there must be a sea-change in practice and a means to control poor prescribing practice should it continue in our hospitals.

\section{Marc Mendelson}

President, Infectious Diseases Society of Southern Africa

President, Federation of Infectious Diseases Societies of Southern Africa

\section{Andrew Whitelaw}

Chairperson, Infection Control Society of Southern Africa

\section{Mark Nicol}

Chairperson, South African Society for Clinical Microbiology

Adrian Brink

Co-Chair, South African Antibiotic Stewardship Programme

\section{References}

1. Communicable Diseases Communique. Outbreak of newly emerged, highly antibiotic resistant bacteria in hospitalized patients in Gauteng Province: New Delhi metallo-beta-lactamase (NDM-1). NICD Communique 2011;10(10):2-3.

2. Brink AJ, Coetzee J, Clay C, et al. Emergence of New Delhi metallo-beta-lactamase (NDM-1) and Klebsiella pneumoniae carbapenemase (KPC-2) in South Africa. J Clin Microbiol 2012;50:525-527. [PMID: 22116157]

3. Shlaes D, Gerding D, John Jr J, et al. Society for Healthcare Epidemiology of America and Infectious Diseases Society of America joint committee on the prevention of antimicrobial resistance: Guidelines for the prevention of antimicrobial resistance in hospitals. Clin Infect Dis 1997;25:584-599. [PMID: 9314444]

4. Hassan M, Tuckman HP, Patrick RH, Kountz DS, Kohn JL. Cost of hospital-acquired infection. Hosp Top 2010;88(3):82-89. [PMID: 20805070]

5. Denton M, Kerr K, Mooney L, et al. Transmission of colistin-resistant Pseudomonas aeruginosa between patients attending a pediatric cystic fibrosis center. Pediatr Pulmonol 2002;34(4):257-261. (PMID: 12205566

6. Falgas ME, Rafilidis PI, Matthaiou DK, et al. Pandrug-resistant Klebsiella pneumoniae, Pseudomonas aeruginosa and Acinetobacter baumannii infections: characteristics and outcome in a series of 28 patients. Int J Antimicrob Agents 2008;32:450-454. [PMID:18768302]

7. Lee JY, Lim MH, Heo ST, Ko KS. Repeated isolation of Pseudomonas aeruginosa isolates resistant to both polymixins and carbapenems from 1 patient. Diagn Microbiol Infect Dis 2012;72(3):267-271. [PMID: 22217703]

8. Brink A, Coetzee J, Clay C, et al. The spread of carbapenem-resistant Enterobacteriaceae (CRE) in South Africa: Risk factors for acquisition and prevention. S Afr Med J 2012;102(7):599-601 (this issue). . Best Care....Always! http://www.bestcare.org.za (accessed 19 February 2012).

. Visser A, Moore DP, Whitelaw A, et al. GARP: Part VII. Interventions. S Afr Med J 2011;101(8, Pt 2):587-595.

1. MacDougall C, Polk RE. Antimicrobial stewardship programs in health care systems. Clin Microbiol Rev 2005;18:638-656. [PMID: 16223951] 